\title{
Pharmacogenetic Applications and Pharmacogenomic Approaches in Schizophrenia
}

\author{
M. J. Arranz • V. Perez · J. Perez • B. Gutierrez • \\ A. Hervas
}

Published online: 11 January 2013

C Springer Science + Business Media New York 2013

\begin{abstract}
Several genetic factors have been identified that, in combination with clinical and environmental factors, contribute to the variability in response to treatment with antipsychotic drugs. Functional polymorphisms in genes coding for cytochrome-P450 (CYP) enzymes, responsible for the metabolism of over $85 \%$ of drugs, have been associated with the development of drug-induced side-effects, and functional polymorphisms in dopamine and serotonin genes may be associated with adverse reactions and treatment efficacy. Recent estimations have suggested that selecting the drug and clinical dose according to the patient's genetic profile may result in a significant improvement of treatment efficacy (10-15\%) and safety (15-20\%). This information has prompted the development of commercial kits to facilitate the clinical application of pharmacogenetic information. However, prospective studies confirming the
\end{abstract}

M. J. Arranz ( $\square)$

Fundació Docència i Recerca Mutua Terrassa, Hospital

Universitari Mutua Terrassa, University of Barcelona,

c/Sant Antoni, 19, 08221 Terrassa, Spain

e-mail: maria.arranz@kcl.ac.uk

M. J. Arranz · V. Perez - J. Perez

Department of Psychiatry, CIBERSAM, Hospital Santa

Creu i Sant Pau, Barcelona, Spain

M. J. Arranz

Department of Neuroscience, Institute of Psychiatry,

King's College London, London, UK

B. Gutierrez

Department of Psychiatry, CIBERSAM, University of Granada, Granada, Spain

A. Hervas

Department of Child Psychiatry, Hospital Universitari Mutua

Terrassa, University of Barcelona, Terrassa, Spain clinical and economical benefits of these tests are required before their widespread implementation in clinical practice.

Keywords Pharmacogenetics - Antipsychotics · CYP . Dopamine $\cdot$ Serotonin $\cdot$ Schizophrenia

\section{Introduction}

Schizophrenia, schizoaffective and related disorders are common human disorders, with severe detrimental effects on patients and their caretakers. Treatment of schizophrenia disorders is still far from satisfactory, with up to $30-50 \%$ of patients not responding adequately to pharmacological treatments. Additionally, the antipsychotic drugs used for the treatment of psychotic symptoms induce a number of severe and long-lasting effects. First-generation antipsychotics (FGA, also known as classical or typical antipsychotics), characterised for their strong affinity for dopaminergic receptors, are known to cause extra-pyramidal side-effects (i.e., akathisias and dyskinesias) which may remain after treatment withdrawal. Second-generation or atypical antipsychotics (SGA) have a lower incidence of EPS, but cause other important and severe side-effects (weight gain, diabetes, hypertension, obesity and dyslipidemia amongst others) [1]. Treatment failure and side effects are thought to be caused by a combination of clinical, environmental and genetic factors. Knowledge of these influencing factors may help to improve psychopharmacological treatment and prevent adverse reactions.

Pharmacogenetic studies have proved that antipsychotic treatment variability has an important degree of heritability, although the later has not been quantified through systematic twin studies. Nevertheless, concordance in the response to antipsychotic treatment has been reported in a number of 
twin pairs suggesting a strong genetic contribution [2]. However, it is clear that genetic factors can only partly explain treatment variability, and that clinical and environmental factors have an important contribution to antipsychotic response. Clinical observations such as early treatment response and significant weight gain are linked to good prognosis, whereas early age of onset and presence of extra-pyramidal symptoms predict poor response to antipsychotics [3-5]. Caffeine consumption inhibits the activity of CYP1A2, an enzyme involved in the metabolism of the frequently used antipsychotics olanzapine and clozapine, whereas smoking induces CYP1A2 activity, increasing clearance rates [6]. Co-administration of antipsychotics competing for the same enzyme leads to their inhibition [7], reducing treatment efficacy and increasing side-effects. Other non-genetic factors, including demographic (e.g., gender and ethnic group) and social factors (e.g., family or social support) may also play a role in treatment outcome. Detailed reviews on non-genetic factors influencing antipsychotic response can be found in the literature and are not the scope of this review. These non-genetic factors have complicated the identification of genetic components of treatment variability. Unfortunately, the majority of the pharmacogenetic studies conducted to date had limited clinical and environmental information and their contribution could not be assessed along with the influence of genetic factors. Current strategies include the incorporation of detailed clinical and environmental information in pharmacogenetic studies to elucidate the real influence of genetic variants. Nevertheless, a number of genes containing potentially influential variants have been identified.

Pharmacogenetic (selected candidate genes) and pharmacogenomic (genome-wide investigations) strategies have been used to identify genetic factors related to antipsychotic treatment variability. While most of the findings currently translated into clinical practice have been obtained via pharmacogenetic strategies, pharmacogenomic strategies interrogating the whole genome have revealed novel areas of interest for antipsychotic activity [8]. However, due to the difficulty in collating samples large enough to achieve reliable statistical power, the results of pharmacogenomic studies on antipsychotics need to be confirmed before their implementation in clinical practice and will not be detailed in this review. The following sections will summarise the most significant pharmacogenetic findings and will give an overview of their potential clinical applications.

\section{Pharmacogenetics of Drug Metabolism and Transport}

The existence of functional polymorphisms in genes coding for phase I metabolic enzymes has been known since the 1950s, when the effect of one such CYP2D6 polymorphism on debrisoquine metabolism was discovered. Since then a number of functional genetic variants that alter the metabolic activity of enzymes to poor (PM), intermediate (IM) or ultrarapid (UM) have been described in several enzymes of the hepatic cytochrome P450 (P450). These enzymes are responsible for the metabolism of more than $85 \%$ of xenobiotics, including most psychotropic drugs. Table 1 includes a summary of currently available antipsychotics and their main metabolic pathways. The presence of these functional polymorphisms has been directly related to drug clearance [9], with PM variants associated with higher plasma levels and the UM forms with rapid clearance and lower plasma concentrations of drugs or their metabolites. The work of Kirchheiner and collaborators illustrated the influence of these polymorphisms on antipsychotic dose requirements [10]. The authors showed that individuals who were CYP2D6 or CYP2C19 PMs required significantly lower doses of their substrate antidepressant or antipsychotic drugs than normal extensive metabolizers. In contrast, UM individuals required an increment of up to threefold normal clinical doses to acquire the drug/metabolite plasma levels associated with therapeutic response. Patients treated with antipsychotics with narrow dose ranges are at higher risk of developing movement disorders when carrying copies of PM variants, whereas UM patients are more likely to fail to respond to standard doses of FGA and SGA and may require higher dosages. Interestingly, these polymorphisms display varying frequencies amongst ethnic groups. For example, CYP2D6 individuals are relatively frequent in European Caucasian populations (7-10\%) and relatively infrequent in Asian populations (1-2\%) [11]. Significant geographical variation is also common in other CYP functional polymorphisms and may partially explain the differences in treatment response observed between populations.

Pharmacogenetic studies provide further evidence of the importance of CYP variants on antipsychotic treatment outcome. CYP2D6, CYP1A2 and CYP17A1 functional variants have been related to risk of developing tardive dyskinesia (TD) [12-15] and weight gain [16, 17], although

Table 1 List of main metabolic pathways used to process commonly used antipsychotic drugs

\begin{tabular}{lll}
\hline Drug & Type & Metabolic pathway \\
\hline Aripiprazole & SGA & CYP2D6, CYP3A \\
Clorpromazine & FGA & CYP2D6, CYP1A2 \\
Haloperidol & FGA & CYP2S6, CYP3A, CYP1A2 \\
Olanzapine & SGA & CYP1A2, CYP2D6 \\
Risperidone & SGA & CYP2D6, CYP3A \\
Clozapine & SGA & CYP1A2, CYP3A, CYP2D6, CYP2C19
\end{tabular}

$F G A$ first generation antipsychotic, $S G A$ second generation antipsychotic 
findings are not universally replicated [8]. For instance, a study of CYP mutations in a large cohort of patients treated with antipsychotics (the CATIE study) failed to find correlations with clinical outcome [18]. Replication failure is often the result of differences in clinical and environmental characteristics between independent studies [19]. The CATIE study comprised patients treated with a variety of FGA and SGA which may have confounded the analyses. Nevertheless, a recent reanalyses of the study data, stratifying by type of treatment, revealed associations between CYP3A43 polymorphisms and response to olanzapine [20]. Additionally, meta-analyses of CYP findings including positive and negative reports confirm their contribution to adverse reactions [21], although their contribution to treatment efficacy is yet unconfirmed.

Relatively few studies have investigated the contribution of conjugation enzymes (phase II enzymes) on treatment response and no clear association has been reported [8]. However, the number of studies investigating phase II enzymes is relatively modest and further research is required to elucidate their contribution to treatment variability. Finally, antipsychotic drugs display varying degrees of affinity for the P-glycoprotein (PgP, coded by the $A B C B 1$ gene, [22]), a transmembrane transporter in the blood-brain barrier. Recent studies have shown that $A B C B 1$ 3435-T allele and ABCB1 2667-T/3435-T haplotype carriers were more frequent among subjects without extrapyramidal syndromes [23], suggesting that this transporter protein, involved in brain clearance of antipsychotics, may also contribute to treatment variability.

\section{Pharmacogenetics of Treatment Response}

Pharmacogenetic studies on the level of efficacy of antipsychotic treatments have been marred by the difficulty of accurately assessing treatment response and the differing criteria used [8]. Thus, replication failure is common, and contradicting reports are often the norm. Nevertheless, pharmacogenetic studies have confirmed the clinical validity of targeting dopamine and serotonin systems, and shed light on other genes contributing to antipsychotic efficacy.

An average of 60-80\% occupancy of brain dopamine receptors has been linked with therapeutic efficacy, and higher levels of occupancy lead to development of sideeffects [24]. All currently available antipsychotics block dopamine receptors to a varying degree. FGA display strong affinity whereas SGA display moderate to high affinity for D2, D3 and D4 receptors. Numerous pharmacogenetic studies confirm the relevance of dopamine receptors for antipsychotic efficacy [2,8]. Figure 1 summarises the number of published reports associating

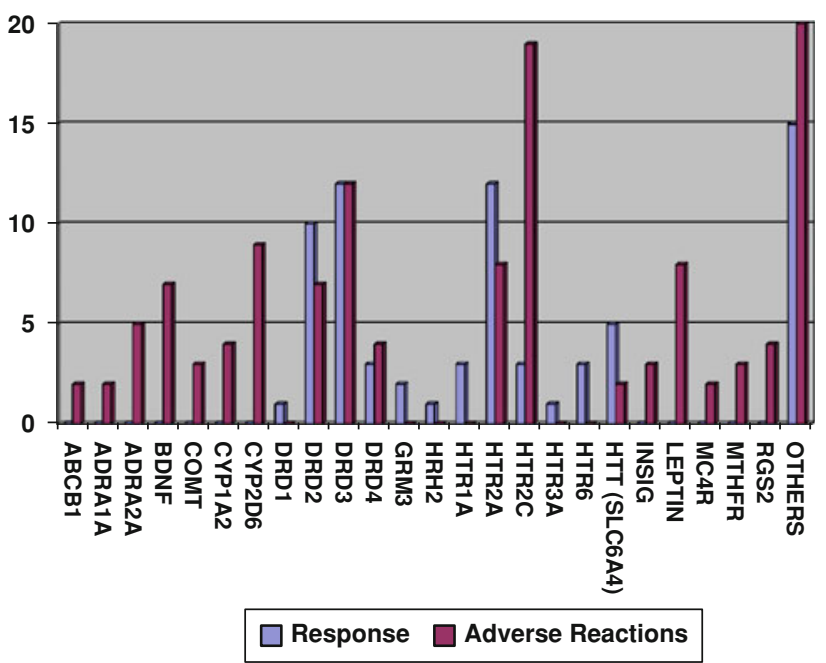

Fig. 1 Approximated numbers of published pharmacogenetic studies reporting statistically significant genetic associations with response and adverse reactions

genetic variants (grouped by gene) with level of antipsychotic efficacy. The most significant findings associate a D2 -141-C Ins/Del polymorphism with antipsychotic response, with patients carrying the $-141-\mathrm{C}$ Del variant, associated with lower expression of the receptor protein, presenting poorer response to treatment [25]. Similarly, a D3 Ser9Gly polymorphism has been reported to contribute to treatment variability, with carriers of the high-dopamine affinity Ser9 variant presenting better treatment response [26]. Less consisting findings associate other dopamine variants with treatment efficacy [8].

There is strong evidence supporting the contribution of serotonergic variants to treatment variability. A 5-HT2A promoter region variant, $-1438-\mathrm{G}$, associated with lower expression of the receptor protein [27], and a strongly linked synonymous allele, 102-C, are increased in patients not responding to the SGA clozapine, risperidone and olanzapine $[8,28]$. Similarly, a 5-HT2A 452Tyr variant, with reduced ability to activate phospholipases $\mathrm{C}$ and $\mathrm{D}$ [29], is reportedly more frequent in non-responding patients. Polymorphic variants in 5-HT1A, 5-HT2C, 5-HT3A and 5-HT6 also contribute to drug efficacy [30-37], reinforcing the relevance of the serotonergic system in antipsychotic activity.

Minor contribution of genetic variants in other targeted neurotransmitter systems has been reported, including reports of association between polymorphisms in glutamatergic (GRM3) [38] and histaminic (H2, H3) [31, 39] receptor genes. This evidence suggests that these systems play a minor role in the mechanism of action of currently available antipsychotics. Nevertheless, this does not invalidate the potential therapeutic validity of these and other neurotransmitter systems, which may be the target of 
novel drugs. Finally, a number of polymorphisms in genes related to the aetiology of the disease (ZNF804, DTNBP1, NRG1) [40-43], metabolism of neurotransmitters (BDNF, COMT) [44-46], cellular development, signalling, transport and other functions (AKAP13, CACNA1, GRIA1, GRIA2, GRIA4, GHRL, NRXN1) [43, 47-51] have also been reported in association with response. However, these findings have yet to be confirmed in independent studies.

It is important to note that none of the associations described in this section have been widely replicated. Phenotype variability and environmental influences are confounding factors that interfere with the identification of genetic associations both related to drug metabolism and drug response and induce false negative and false positive results. An alternative reason behind replication failure of response findings is the use of indirect markers. Many of the response-associated polymorphisms do not have a significant functional effect, and may act as indirect indicators of causal polymorphisms to which they are linked. The linkage status may vary in different populations and thus the magnitude and/or the direction of the associations may change. Additionally, the genetic effects observed are moderate to low (odds ratios generally below 2), inferior to the values observed in the associations with functional metabolic polymorphisms. Treatment response is a complex phenotype that may be determined, in addition to environmental factors, by several contributing genes from drug targets and interacting proteins, in contrast with drug metabolism which is mainly determined by enzymatic activity. Therefore it is not surprising that the associations are not as clear, which diminishes their clinical value and applicability. Nevertheless, these findings provide important information on the therapeutic value of currentlyavailable drug targets and may help in the design of improved novel antipsychotics.

\section{Pharmacogenetics of Antipsychotic-Induced Side-Effects}

FGA and SGA produce important and long-lasting sideeffects with negative effects both in terms of prognosis and treatment compliance. Adverse reaction phenotypes are relatively easy to determine, which facilitates the identification of related genetic factors.

Weight gain and other effects contributing to metabolic syndrome (obesity, diabetes, hypertension, and cardiovascular events) are considered the most important antipsychoticinduced side-effects, and are observed in up to $57 \%$ patients treated with SGA, and $17 \%$ treated with FGA. Serotonin regulates feeding behaviour and alterations in serotonin transmission have been suggested to contribute to antipsychotic-induced weight gain. Several studies associating polymorphisms in serotonin receptors with weight gain support this hypothesis. The most significant finding associates a 5-HT2C gene polymorphism, -759-T/C, with weight-gain and BMI, although the direction of the association varies in different studies [17, 52, 53]. A meta-analysis confirmed the protective effects of the -759-T allele against weight gain in Caucasian patients [54]. Several reports implicate genes involved in energy intake and expenditure, namely leptin (LEP) [55-58] and melanocortin receptor 4 (MC4R) [59, 60], in antipsychotic induced weight gain, thus suggesting a multigenic cause of this effect. Other energy regulatory genes, including INSIG2 and GHRL have also been associated with weight gain in unconfirmed reports $[49,61]$.

TD is a severe and long-lasting side-effect associated mainly with the use of FGA, and to a minor extent with some SGA (i.e., risperidone). Patients presenting CYP2D6 PM alleles and D2 variants associated with lower receptor expression, resulting in higher receptor occupancy, are at higher risk of developing TD [25, 62, 63]. 5-HT2C and 5-HT2A low expression variants have also been associated with TD development, especially when considering the age of the patient, probably reflecting an indirect influence on dopamine regulation [64-66].

The genetic effects observed in these studies are moderate to high, suggesting a potential clinical applicability. However, confirmation of the direction and predictive value of the findings in different population groups is required before the clinical application of this information. Interestingly, the number of genes associated with the development of sideeffects surpasses the number of genes associated with treatment efficacy (see Fig. 1), reinforcing the need for novel drugs directed to targets of proven clinical value.

\section{Pharmacogenetic Applications}

The ultimate goal of pharmacogenetic research is the use of genetic information for the personalisation of treatment. However, few of the reported findings have been translated into clinical applications in spite of their potential benefits.

The implications of CYP findings for antipsychotic treatment are clear. Characterising the genetically-determined CYP status of the patients before the start of the treatment may provide useful and beneficial information. Estimations of the clinical and economical benefits of CYP genetic testing support their implementation in clinical practice. It has been suggested that the adjustment of clinical doses according to the individuals' genetic profile may result in a 10-15\% improvement in the level of efficacy and in 15-20\% reductions in side-effects. Unfortunately, this information is not yet widely used in clinical practice. The clinical value of genetic information on drug targets is not yet clearly established. Most of the reported associations with 
drug target variants have a limited predictive value and cannot be used as single-markers of response or side-effects. Although attempts have been made at combining information in several genes to improve prediction values, further research on their clinical validity is required.

Several tests for the genetic determination of the metabolic status of patients, prediction of the level of efficacy, and risk of developing side-effects are already available in commercial and clinical laboratories (see Table 2 for summary list). CYP functional polymorphisms aside, most of these tests are of limited predictive value, never reaching $100 \%$ certainty (and far from it). This is not surprising given that the majority of the tests do not consider the clinical and environmental factors that play a role in treatment variability, and include unconfirmed genetic findings. Nevertheless, the information contained in these tests has the potential to improve treatment response by helping to select the most beneficial treatment at the right dose for patients according to their genetic predisposition. In spite of this, the use of genetic tests to assist drug selection in psychiatry is minimal. Lack of information and limited access to clinical or reference laboratories with capabilities for pharmacogenetic testing are partly to blame. However, the main reason that may hinder the use of pharmacogenetic tests is the lack of supporting research assessing the benefits. To date, no study has investigated if the adjustment of clinical doses according to

Table 2 List of available pharmacogenetic tests for selected antipsychotic drugs

\begin{tabular}{|c|c|c|}
\hline Test & Information provided & Manufactured \\
\hline Amplichip & $\begin{array}{l}\text { CYP2D6 and CYP2C19 } \\
\text { polymorphisms }\end{array}$ & Roche \\
\hline $\begin{array}{l}\text { Tm Tag-it } \\
\text { Mutation } \\
\text { detection kit }\end{array}$ & $\begin{array}{l}\text { CYP2D6 and CYP2C19 } \\
\text { most common } \\
\text { polymorphisms }\end{array}$ & $\begin{array}{l}\text { TMBIOSCIENCES- } \\
\text { Luminex }\end{array}$ \\
\hline $\begin{array}{l}\text { Vercode } \\
\text { ADME core } \\
\text { panel }\end{array}$ & $\begin{array}{l}\text { CYP450 enzymes and } \\
\text { polymorphisms in other } \\
\text { metabolic enzymes }\end{array}$ & Illumina \\
\hline $\begin{array}{l}\text { DMET Plus } \\
\text { premier pack }\end{array}$ & $\begin{array}{l}1,936 \text { markers in } \\
\text { metabolizing genes }\end{array}$ & Affymetrix \\
\hline Genecept assay & $\begin{array}{l}\text { Polymorphisms CYP2D6, } \\
\text { CYP2C19, SLC6A4, } \\
\text { DRD2, AnkirynG, COMT } \\
\text { and MTHFR genes }\end{array}$ & Genomind \\
\hline GeneSightRx & $\begin{array}{l}\text { Polymorphisms in CYP2D6, } \\
\text { CYP2C19, CYP2C9, } \\
\text { CYP1A2, SLC6A4 and } \\
\text { HTR2A genes }\end{array}$ & Assure Rx \\
\hline BrainChip & $\begin{array}{l}\text { Polymorphisms in CYP } \\
\text { enzymes and drug target } \\
\text { genes }\end{array}$ & Brainco \\
\hline Neurofarmagen & $\begin{array}{l}\text { Polymorphisms in CYP } \\
\text { enzymes and drug target } \\
\text { genes }\end{array}$ & AB Biotics \\
\hline
\end{tabular}

the patient's CYP genetic variants results in a reduction of the incidence of side-effects, and in an improvement of response. Similarly, no prospective study has proved that the use of pharmacogenetic prediction tests for the selection of drug type positively influences the level of efficacy that is reflected in a reduction of hospitalization time, improvement of social functioning, etc. Thus, there is little supporting evidence encouraging the use of pharmacogenetic information in clinical settings. Without a prospective trial to prove the clinical and economical benefits of using genetic information to aid drug and dose selection, clinicians are right to doubt the benefits of a pharmacogenetic approach. The affluence of commercial tests offering a variety of genetic information, sometimes poorly translated into clinically useful information, reinforces the need for prospective validating studies.

\section{Conclusion}

Pharmacogenetic and Pharmacogenomic studies have identified several genetic factors that, in combination with clinical and environmental events, contribute to the variability in response to treatment with antipsychotic drugs. Functional genetic variants in CYP metabolising enzymes are the most promising findings, with the potential to improve drug safety and efficacy. Findings in drug targets require further research, but may be valuable for the risk evaluation of side-effects. Findings relating genetic variants with treatment response have a limited predictive and clinical value, especially in absence of environmental and clinical information. In spite of the supporting evidence, pharmacogenetic testing is not widely used in psychiatric practice. Prospective studies evaluating the clinical and economical benefits may convince the clinicians of the value of pharmacogenetic information for the improvement of antipsychotic efficacy and safety.

Future research in the field will investigate the influence of clinical and environmental factors, along with genetic factors, to better determine their contribution to antipsychotic efficacy. Genomic and epigenetic studies may also reveal novel genetic areas of interest for antipsychotic activity.

Acknowledgments We thank Olga Baeza and Dolors Estellers for their useful comments and support during the writing of this manuscript.

\section{References}

1. Miyamoto S, Duncan GE, Marx CE, Lieberman JA. Treatments for schizophrenia: a critical review of pharmacology and mechanisms of action of antipsychotic drugs. Mol Psychiatry. 2005;10:79. 
2. Arranz MJ, Munro JC. Toward understanding genetic risk for differential antipsychotic response in individuals with schizophrenia. Expert Rev Clin Pharmacol. 2011;4:389.

3. Stauffer VL, et al. Early response to antipsychotic therapy as a clinical marker of subsequent response in the treatment of patients with first-episode psychosis. Psychiatry Res. 2011; 187:42.

4. Leucht S, Busch R, Kissling W, Kane JM. Early prediction of antipsychotic nonresponse among patients with schizophrenia. J Clin Psychiatry. 2007;68:352.

5. Lieberman JA, et al. Factors influencing treatment response and outcome of first-episode schizophrenia: implications for understanding the pathophysiology of schizophrenia. J Clin Psychiatry 1996;57 Suppl 9:5.

6. Arranz MJ, de Leon J. Pharmacogenetics and pharmacogenomics of schizophrenia: a review of last decade of research. Mol Psychiatry. 2007;12:707.

7. Azuma $J$, et al. The relationship between clinical pharmacokinetics of aripiprazole and CYP2D6 genetic polymorphism: effects of CYP enzyme inhibition by coadministration of paroxetine or fluvoxamine. Eur J Clin Pharmacol. 2012;68:29.

8. Arranz MJ, Rivera M, Munro JC. Pharmacogenetics of response to antipsychotics in patients with schizophrenia. CNS Drugs. 2011;25:933.

9. Teh LK, Bertilsson L. Pharmacogenomics of CYP2D6: molecular genetics, interethnic differences and clinical importance. Drug Metab Pharmacokinet. 2012;27:55.

10. Kirchheiner J, et al. Influence of CYP2C9 and CYP2D6 polymorphisms on the pharmacokinetics of nateglinide in genotyped healthy volunteers. Clin Pharmacokinet. 2004;43:267.

11. Polimanti R, Piacentini S, Manfellotto D, Fuciarelli M. Human genetic variation of CYP450 superfamily: analysis of functional diversity in worldwide populations. Pharmacogenomics. 2012;13: 1951.

12. Segman RH, et al. Interactive effect of cytochrome $\mathbf{P} 450$ 17alpha-hydroxylase and dopamine D3 receptor gene polymorphisms on abnormal involuntary movements in chronic schizophrenia. Biol Psychiatry. 2002;51:261.

13. Basile VS, et al. A functional polymorphism of the cytochrome P450 1A2 (CYP1A2) gene: association with tardive dyskinesia in schizophrenia. Mol Psychiatry. 2000;5:410.

14. Fu Y, et al. Association of CYP2D6 and CYP1A2 gene polymorphism with tardive dyskinesia in Chinese schizophrenic patients. Acta Pharmacol Sin. 2006;27:328.

15. Lucire Y, Crotty C. Antidepressant-induced akathisia-related homicides associated with diminishing mutations in metabolizing genes of the CYP450 family. Pharmgenomics Pers Med. 2011;4:65.

16. Ellingrod VL, Miller D, Schultz SK, Wehring H, Arndt S. CYP2D6 polymorphisms and atypical antipsychotic weight gain. Psychiatr Genet. 2002;12:55.

17. Lane HY, et al. Risperidone-related weight gain: genetic and nongenetic predictors. J Clin Psychopharmacol. 2006;26:128.

18. Grossman I, et al. Genetic determinants of variable metabolism have little impact on the clinical use of leading antipsychotics in the CATIE study. Genet Med. 2008;10:720.

19. Arranz MJ, Munro J, Osborne S, Collier D, Kerwin RW. Difficulties in replication of results. Lancet. 2000;356:1359.

20. Bigos KL, et al. Genetic variation in CYP3A43 explains racial difference in olanzapine clearance. Mol Psychiatry. 2011;16:620.

21. Fleeman N, et al. Cytochrome P450 testing for prescribing antipsychotics in adults with schizophrenia: systematic review and meta-analyses. Pharmacogenomics J. 2011;11:1.

22. Moons T, de Roo M, Claes S, Dom G. Relationship between P-glycoprotein and second-generation antipsychotics. Pharmacogenomics. 2011;12:1193.
23. Jovanović N, et al. The role of CYP2D6 and ABCB1 pharmacogenetics in drug-naïve patients with first-episode schizophrenia treated with risperidone. Eur J Clin Pharmacol. 2010;66:1109.

24. Remington $\mathrm{G}$, et al. A PET study evaluating dopamine D2 receptor occupancy for long-acting injectable risperidone. Am J Psychiatry. 2006;163:396.

25. Zai CC, et al. Meta-analysis of two dopamine D2 receptor gene polymorphisms with tardive dyskinesia in schizophrenia patients. Mol Psychiatry. 2007;12:794.

26. Bakker PR, van Harten PN, van Os J. Antipsychotic-induced tardive dyskinesia and the Ser9Gly polymorphism in the DRD3 gene: a meta analysis. Schizophr Res. 2006;83:185.

27. Parsons MJ, D'Souza UM, Arranz MJ, Kerwin RW, Makoff AJ. The $-1438 \mathrm{~A} / \mathrm{G}$ polymorphism in the 5-hydroxytryptamine type $2 \mathrm{~A}$ receptor gene affects promoter activity. Biol Psychiatry. 2004;56:406.

28. Arranz MJ, et al. Meta-analysis of studies on genetic variation in 5-HT2A receptors and clozapine response. Schizophr Res. 1998; 32:93.

29. Hazelwood LA, Sanders-Bush E. His452Tyr polymorphism in the human 5-HT2A receptor destabilizes the signaling conformation. Mol Pharmacol. 2004;66:1293.

30. Sodhi MS, et al. Association between clozapine response and allelic variation in the 5-HT2C receptor gene. NeuroReport. 1995;7:169.

31. Arranz MJ, et al. Pharmacogenetic prediction of clozapine response. Lancet. 2000;355:1615.

32. Yu YW, et al. Serotonin-6 receptor variant (C267T) and clinical response to clozapine. NeuroReport. 1999;10:1231.

33. Wang L, et al. The $-1019 \mathrm{C} / \mathrm{G}$ polymorphism of the 5-HT(1)A receptor gene is associated with negative symptom response to risperidone treatment in schizophrenia patients. J Psychopharmacol. 2008;22:904.

34. Liu BC, et al. HTR2C promoter polymorphisms are associated with risperidone efficacy in Chinese female patients. Pharmacogenomics. 2010;11:685.

35. Gu B, et al. Association between a polymorphism of the HTR3A gene and therapeutic response to risperidone treatment in drugnaive Chinese schizophrenia patients. Pharmacogenet Genomics. 2008;18:721.

36. Lane HY, et al. Risperidone response and 5-HT6 receptor gene variance: genetic association analysis with adjustment for nongenetic confounders. Schizophr Res. 2004;67:63.

37. Crisafulli $\mathrm{C}$, et al. Case-control association study for 10 genes in patients with schizophrenia: influence of 5HTR1A variation rs 10042486 on schizophrenia and response to antipsychotics. Eur Arch Psychiatry Clin Neurosci. 2012;262:199.

38. Bishop JR, Ellingrod VL, Moline J, Miller D. Association between the polymorphic GRM3 gene and negative symptom improvement during olanzapine treatment. Schizophr Res. 2005;77:253.

39. Wei Z, et al. A pharmacogenetic study of risperidone on histamine $\mathrm{H} 3$ receptor gene (HRH3) in Chinese Han schizophrenia patients. J Psychopharmacol. 2012;26:813.

40. Zuo L, et al. The efficacies of clozapine and haloperidol in refractory schizophrenia are related to DTNBP1 variation. Pharmacogenet Genomics. 2009;19:437.

41. Zhang J, et al. Association analysis of ZNF804A (zinc finger protein 804A) rs1344706 with therapeutic response to atypical antipsychotics in first-episode Chinese patients with schizophrenia. Compr Psychiatry. 2012;53:1044.

42. Mössner R, et al. The schizophrenia risk gene ZNF804A influences the antipsychotic response of positive schizophrenia symptoms. Eur Arch Psychiatry Clin Neurosci. 2012;262:193.

43. Drago A, et al. AKAP13, CACNA1, GRIK4 and GRIA1 genetic variations may be associated with haloperidol efficacy during acute treatment. Eur Neuropsychopharmacol. 2012 (in press). 
44. Weickert TW, et al. Catechol-O-methyltransferase val108/ 158met genotype predicts working memory response to antipsychotic medications. Biol Psychiatry. 2004;56:677.

45. Molero P, Ortuño F, Zalacain M, Patiño-García A. Clinical involvement of catechol-O-methyltransferase polymorphisms in schizophrenia spectrum disorders: influence on the severity of psychotic symptoms and on the response to neuroleptic treatment. Pharmacogenomics J. 2007;7:418.

46. Zai GC, et al. The role of brain-derived neurotrophic factor (BDNF) gene variants in antipsychotic response and antipsychotic-induced weight gain. Prog Neuropsychopharmacol Biol Psychiatry. 2012;39:96.

47. Giegling I, et al. Glutamatergic gene variants impact the clinical profile of efficacy and side effects of haloperidol. Pharmacogenet Genomics. 2011;21:206.

48. Crisafulli $\mathrm{C}$, et al. Influence of GRIA1, GRIA2 and GRIA4 polymorphisms on diagnosis and response to antipsychotic treatment in patients with schizophrenia. Neurosci Lett. 2012;506:170.

49. Yang Y, et al. Association between ghrelin gene (GHRL) polymorphisms and clinical response to atypical antipsychotic drugs in Han Chinese schizophrenia patients. Behav Brain Funct. 2012; 8:11.

50. Lett TA, et al. The putative functional rs1045881 marker of neurexin-1 in schizophrenia and clozapine response. Schizophr Res. 2011;132:121.

51. Souza RP, Meltzer HY, Lieberman JA, Le Foll B, Kennedy JL. Influence of neurexin 1 (NRXN1) polymorphisms in clozapine response. Hum Psychopharmacol. 2010;25:582.

52. Zhang ZJ, Zhang XB, Sha WW, Reynolds GP. Association of a polymorphism in the promoter region of the serotonin 5-HT2C receptor gene with tardive dyskinesia in patients with schizophrenia. Mol Psychiatry. 2002;7:670.

53. Miller DD, Ellingrod VL, Holman TL, Buckley PF, Arndt S. Clozapine-induced weight gain associated with the 5HT2C receptor $-759 \mathrm{C} / \mathrm{T}$ polymorphism. Am J Med Genet B Neuropsychiatr Genet. 2005;133B:97.

54. Sicard MN, et al. Polymorphisms of the HTR2C gene and antipsychotic-induced weight gain: an update and meta-analysis. Pharmacogenomics. 2010;11:1561.

55. Brandl EJ, et al. Association study of polymorphisms in leptin and leptin receptor genes with antipsychotic-induced body weight gain. Prog Neuropsychopharmacol Biol Psychiatry. 2012;38:134.
56. Zhang XY, et al. Association of clozapine-induced weight gain with a polymorphism in the leptin promoter region in patients with chronic schizophrenia in a Chinese population. J Clin Psychopharmacol. 2007;27:246.

57. Calarge CA, et al. Leptin gene $-2548 \mathrm{G} / \mathrm{A}$ variants predict risperidone-associated weight gain in children and adolescents. Psychiatr Genet. 2009;19:320.

58. Srivastava V, Deshpande SN, Nimgaonkar VL, Lerer B, Thelma B. Genetic correlates of olanzapine-induced weight gain in schizophrenia subjects from north India: role of metabolic pathway genes. Pharmacogenomics. 2008;9:1055.

59. Chowdhury NI, et al. Genetic association study between antipsychotic-induced weight gain and the melanocortin-4 receptor gene. Pharmacogenomics J. 2012 (in press).

60. Malhotra AK, et al. Association between common variants near the melanocortin 4 receptor gene and severe antipsychotic druginduced weight gain. Arch Gen Psychiatry. 2012;69:904.

61. Le Hellard S, et al. Association between the insulin-induced gene 2 (INSIG2) and weight gain in a German sample of antipsychotictreated schizophrenic patients: perturbation of SREBP-controlled lipogenesis in drug-related metabolic adverse effects? Mol Psychiatry. 2009;14:308.

62. Lee HJ, Kang SG. Genetics of tardive dyskinesia. Int Rev Neurobiol. 2011;98:231.

63. Koning JP, et al. Association of two DRD2 gene polymorphisms with acute and tardive antipsychotic-induced movement disorders in young Caucasian patients. Psychopharmacology. 2012; 219:727.

64. Hsieh CJ, Chen YC, Lai MS, Hong CJ, Chien KL. Genetic variability in serotonin receptor and transporter genes may influence risk for tardive dyskinesia in chronic schizophrenia. Psychiatry Res. 2011;188:175.

65. Lerer B, et al. Combined analysis of 635 patients confirms an age-related association of the serotonin $2 \mathrm{~A}$ receptor gene with tardive dyskinesia and specificity for the non-orofacial subtype. Int J Neuropsychopharmacol. 2005;8:411.

66. Segman RH, et al. Association between the serotonin $2 \mathrm{C}$ receptor gene and tardive dyskinesia in chronic schizophrenia: additive contribution of 5-HT2Cser and DRD3gly alleles to susceptibility. Psychopharmacology. 2000;152:408. 